\title{
INTERACCIÓN AGUA-SEDIMENTOS-TRAZADOR FLUORESCENTE EN UN REACTOR. MEDICIÓN DE FLÓCULOS
}

\author{
Alex Wolfenson, Verónica Lanza, Lucas Palman, MatiasImhoff, Ana Alvarez y Alfredo Trento ${ }^{(*)}$ \\ Facultad de Ingeniería y Ciencias Hídricas,Universidad Nacional del Litoral. Santa Fe, Santa Fe, Argentina. \\ ${ }^{(*)}$ e-mail: alfredotrento@gmail.com
}

\begin{abstract}
RESUMEN
El objetivo fue estudiar en condiciones controladas de laboratorio la interacción entre el trazador fluorescente Amidorodamina $\mathrm{G}$ y sedimentos del río Salado(Santa $\mathrm{Fe}$ ), con $\mathrm{d}_{50}=11.6 \mu \mathrm{m}$ y $97 \%$ de sedimentos finos. Se utilizó un reactor cilíndrico diseñado para tal fin, implementándose diferentes condiciones hidrodinámicas. Las velocidades tangenciales y radiales se midieron con una sonda ADV, los tamaños de flóculos, turbiedady otras variables se determinaron con sondas multiparamétricas. El rango de velocidades angulares (N) utilizado fue función de las tensiones de corte esperadas para distintos escenarios del río. Los resultados obtenidos en una primera etapa de ensayosutilizando los sedimentos en agua destilada,se contrastaron con los de una segunda etapa en la que se agregó trazador.Los máximos tamaños de flóculos, $\mathrm{df}_{50} \approx 48 \mu \mathrm{m}$, se obtuvieron sin rodamina, para concentraciones de sólidos suspendidos totales (SST) entre 50 y $100 \mathrm{mg} / \mathrm{L}$ y $\mathrm{N}>150 \mathrm{rpm}$. Para SST mayores a $100 \mathrm{mg} / \mathrm{L}$ los $\mathrm{df}_{50}$ decayeron para todo el rango de $\mathrm{N}$ hasta $\mathrm{df}_{50} \approx 25 \mu \mathrm{m}$, independientemente de la presencia del trazador. Para SST $>150 \mathrm{mg} / \mathrm{L}$ con rodamina los $\mathrm{df}_{50}$ fueron mayores entre 5 y $10 \%$ a los obtenidos sin rodamina. Se concluye que tanto la turbiedad como el tamaño de flóculos fueron afectados por la presencia del trazador, para distintas N y SST.
\end{abstract}

Palabras clave: Trazador fluorescente, sedimentos, reactor

\begin{abstract}
The objective was to study in controlled laboratory conditions the interaction between the Amidorhodamine $\mathrm{G}$ fluorescent tracer and sediments of the Salado river (Santa Fe), with $\mathrm{d} 50=11.6 \mu \mathrm{m}$ and $97 \%$ of fine sediments. A cylindrical reactor designed for this purpose and for different hydrodynamic conditions were used. The tangential and radial velocities were measured by an ADV probe, while floc sizes, turbidity and other variables were determined with multiparametric probes. The range of angular velocities $(\mathrm{N})$ used was a function of the expected shear stresses for different river scenarios. The results obtained in a first test using the sediments in distilled water were contrasted with those of a second test, in which tracer was added. The maximum floc sizes, $\mathrm{df}_{50} \approx 48 \mu \mathrm{m}$, were obtained without rhodamine, for concentrations of total suspended solids (SST) between 50 and $100 \mathrm{mg} / \mathrm{L}$ and $\mathrm{N}>150 \mathrm{rpm}$. For SST greater than $100 \mathrm{mg} / \mathrm{L}$ the df $\mathrm{f}_{50}$ decayed to $\mathrm{df}_{50} \approx 25 \mu \mathrm{m}$ for the entire range of $\mathrm{N}$, regardless of the presence of the tracer. For SST $>150 \mathrm{mg} / \mathrm{L}$ with rhodamine the $\mathrm{df}_{50}$ were greater between 5 and $10 \%$ than those obtained without rhodamine. It is concluded that both the turbidity and floc sizes were affected by the tracer presence, for different $\mathrm{N}$ and SST.
\end{abstract}

Keywords: Fluorescenttracer, sediments, reactor 


\section{INTRODUCCIÓN}

Debido a sus diversas implicancias ambientales, el transporte de sedimentos suspendidos en cursos de agua es un tema de sumo interés en las disciplinas científicas relacionadas a la hidráulica. En particular los sedimentos finos, por sus propiedades físico-químicas, pueden sorber diferentes sustancias orgánicas e inorgánicas presentes en el agua, formar agregados y alterar su calidad. En ese contexto, la floculación de los finos es un factor importante a considerar en cuanto a la disminución de la transparencia del agua, que incide en la penetración de la luz y por lo tanto sobre la biota. La sedimentación de los agregados es más rápida y como pueden poseer metales pesados, bacterias y materia orgánica en general, pueden luego por resuspensión, transformar el lecho en una fuente secundaria de contaminación (Edzwald et al., 1974). La problemática del conocimiento de los complejos fenómenos de transporte de sedimentos suspendidos en cursos de agua ha sido abordada por técnicas que combinan mediciones de campo (hidrodinámicas, batimétricas y sedimentológicas) con simulaciones numéricas. Las mediciones son relevantes para la implementación y calibración de los parámetros de los modelos, no obstante lo cual, algunos son de difícil cuantificación cuando se pretende realizar una buena simulación del transporte de sedimentos. En este caso se requiere conocer el coeficiente de dispersión longitudinal, $\mathrm{D}_{\mathrm{L}}$, para modelos $1 \mathrm{D}$, o para modelos $2 \mathrm{DH}$ los coeficientes de dispersión longitudinal y transversal, $\mathrm{D}_{\mathrm{xx}}$ y $\mathrm{D}_{\mathrm{yy}}$, respectivamente. Estos pueden ser determinados por diferentes métodos: fórmulas empíricas (Fischer, 1968; Liu, 1977, Fischer et al., 1979; Rutherford, 1994), mediciones hidrodinámicas de detalle (Carr et al., 2007; Shen et al., 2010) o mediante experimentos con trazadores (Clark et al., 2009; Hamidifar et al., 2015). Este último método es el más preciso, pero exige cuidadosas y costosas mediciones en campo. Básicamente consiste en inyectar una cantidad de masa conocida de un trazador fluorescente y registrar sus concentraciones a lo largo y ancho del curso de agua con un instrumento adecuado. Se asume que el trazador se disuelve completamente en el agua y se mueve como un constituyente pasivo y conservativo. Esa hipótesis es equivalente a sostener que la dinámica del trazador no está afectada por la presencia de los finos floculados.
Otro método ampliamente difundido en la comunidad científica, para estudiar la dinámica de los sedimentos es el uso de diversos dispositivos de laboratorio, por ejemplo: jarras de floculación, columnas de sedimentación, canales anulares,floculadoresCouette, de disco y reactores (Chapra, 1997). De este modo, es posible lograr aproximaciones de interés científico en laboratorio, para aquellos casos o problemas que resultan difíciles y costosos de estudiar en campo. La complejidad del problema se pone de manifiesto si se considera la diversidad de tamaños de los sedimentos, el movimiento turbulento a distintas escalas espaciales y el problema de la floculación, cuando las propiedades cohesivas son relevantes (Winterwerp, 2002).

Entre los trabajos con reactores de mezcla completa se destacan los de Mikes et al. (2004); Cheng et al. (2008); Bertrand et al. (2012); Benevides et al. (2014);Zhenbei et al. (2018), entre otros. En esos trabajos se trató de representar procesos de transporte de sedimentos bajo condiciones controladas y que guardaran cierta semejanza respecto de parámetros específicos, como por ejemplo las tensiones de corte medias del flujo.Por otra parte, Goeset al. (2019)mostraron, a partir de ensayos de laboratorio, las dificultades para determinar concentraciones utilizando sondas de turbiedad, en mezclas de sedimentos finos y gruesos, en un reactor.

Con el objetivo de estudiar la interacción entre el trazador y los sedimentos finos para diferentes condiciones hidrodinámicas, en este trabajo se utilizóun reactor cilíndrico, con paletas giratorias en la parte inferior, especialmente diseñado de acuerdo a los criterios de Nagata (1975). Se efectuaron experimentos para diferentes velocidades angulares $(\omega)$ y distintas concentraciones de sólidos suspendidos totales (SST), utilizando sedimentos del río Salado (Santa Fe).

En una primera etapa, los sedimentos finos se mezclaron únicamente con agua destilada y en otra, con agua destilada y una concentración predeterminada del trazador fluorescente Amidorodamina G (Acid Red 50, color index 45220), que en adelante se denominará rodamina. Se contó con un difractómetro laser sumergible LISST25X (Sequoia, 2009) para determinar los tamaños medios de flóculos $\left(\mathrm{df}_{50}\right)$ en cada serie de ensayos y una sonda YSI 6600 (2009) para la determinación de la turbiedad (T). 


\section{METODOLOGIA}

\section{Descripción del reactor}

El reactor utilizado para los ensayos (Figura 1) es un tubo cilíndrico de acrílico transparente de 0.40 $\mathrm{cm}$ de espesor, $19.5 \mathrm{~cm}$ de diámetro (D) y $39.5 \mathrm{~cm}$ de altura $(\mathrm{H})$ que se apoya sobre una base de acrílico de $31 \mathrm{~cm}$ de diámetro. El movimiento rotacional es generado por una paleta de acero de $10 \mathrm{~cm}$ de longitud (d) y $2 \mathrm{~cm}$ de alto, la cual se fija al eje del motor mediante un tornillo de ajuste (ver Figura 2). El motor es trifásico, con una potencia de $0.25 \mathrm{HP}$ y regulado por un convertidor compacto de compensación automática, de alimentación monofásica y frecuencia de salida de hasta $400 \mathrm{~Hz}$. Esto permite asignar al motor la frecuencia que el usuario requiera. Para relacionar las frecuencias del convertidor compacto y la velocidad angular $(\omega)$ de las paletas se utilizó un tacómetro digital (Sinometer DT-2234C).

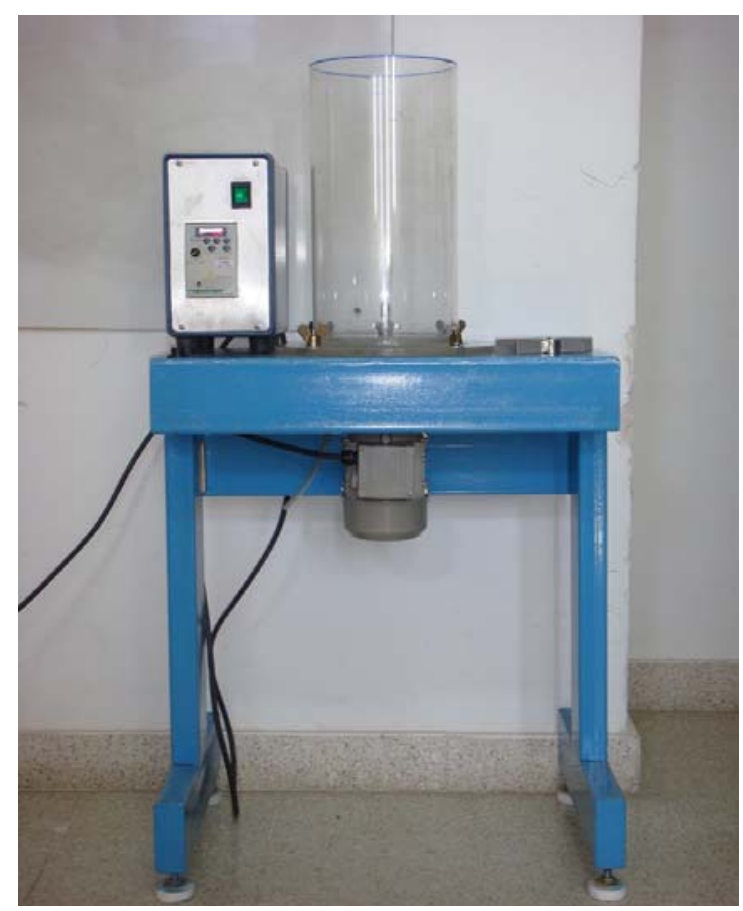

Figura 1.Fotografía del equipo utilizado

\section{Descripción de los equipos de medición}

Para realizar las mediciones se contó con los siguientes instrumentos: FlowTracker (Sontek, 2009) de tecnología acústica Doppler para la medición de velocidades, equipo LISST-25X (Sequoia, 2009) para medir el tamaño de partículas y
Sonda YSI 6600 con sensores de turbiedad y concentración de rodamina (Figura 3).

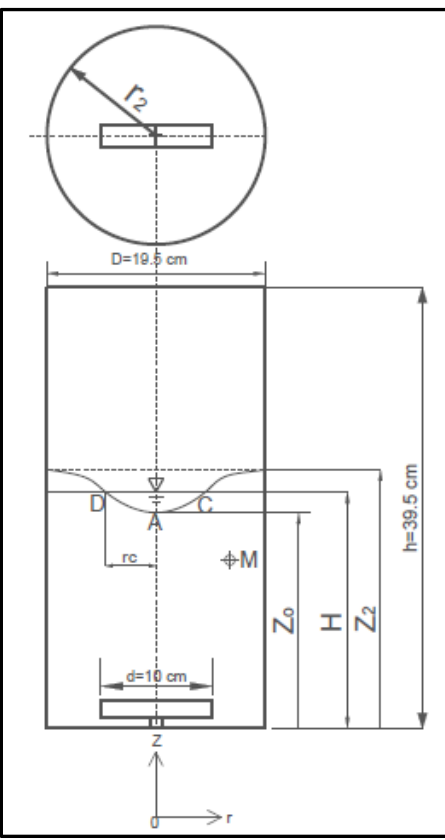

Figura 2.Esquema del reactor con la nomenclatura empleada porNagata (1975)

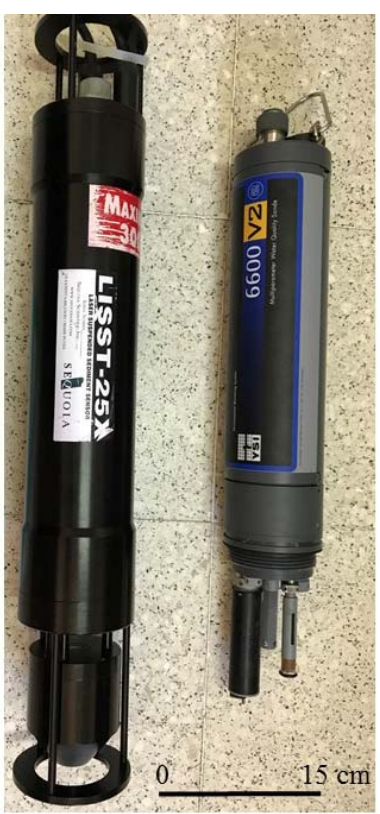

Figura 3. Sonda YSI 6600 a la derecha y sonda LISST-25X a la izquierda de la imagen

La sonda FlowTracker es un velocímetro que utiliza la tecnología $\mathrm{ADV}$, consta de un transmisor que emite señales de sonido, de velocidad y frecuencia conocidas, y dos receptores acústicos a los que llega 
la señal luego de ser reflejada por los objetos en movimiento. El velocímetro requiere la presencia de sólidos en suspensión para obtener valores de velocidad, ya que el equipo no mide la velocidad de la partícula de agua sino del material suspendido que se mueve con ella. Puede medir velocidades en cursos con profundidad mínima de $2 \mathrm{~cm}$, en un rango de 0.0001 a $4.5 \mathrm{~m} / \mathrm{s}$ y con una seguridad de $\pm 1 \%$ de la velocidad medida.

La sonda YSI 6600, para la medición de turbiedad, cuenta con un sensor óptico basado en el principio de refracción; desde un diodo emite un haz de luz, el cual al incidir sobre las partículas cambia su dirección e intensidad. Este cambio es detectado por un fotodetector que transmite la lectura al software Ecowatch, propio de la sonda, el que mediante un algoritmo convierte los registros en unidades de turbidez nefelométricas (NTU), en un rango de 0 a 1000 NTU con una resolución de 0.1 NTU y una seguridad de $\pm 5 \%$ o 2 NTU, aquel que sea mayor.

Para la medición de concentraciones de rodamina el principio de funcionamiento de la sonda YSI 6600 consiste en irradiar, desde un sensor óptico con una longitud de onda particular, el volumen de agua cercano al cabezal; la rodamina al ser irradiada reacciona emitiendo una longitud de onda mayor, que es detectada por el sensor. El intervalo de muestreo $(\Delta t)$ es regulado por el operador, adoptándose un intervalo $\Delta \mathrm{t}=5 \mathrm{~s}$. La sonda puede medir en un rango de $0-200 \mu \mathrm{g} / \mathrm{L}$, con una resolución de $0.1 \mu \mathrm{g} / \mathrm{L}$ y una seguridad de $\pm 1 \mu \mathrm{g} / \mathrm{L}$ o $5 \%$ de la lectura, YSI 6600 (2009).

La sonda LISST-25X es un difractómetro que cuenta con un sensor láser para medir tamaños de sedimentos suspendidos en cuerpos de agua. Fue desarrollada por la empresa Sequoia Inc. LISST es acrónimo de Laser in-situ Scattering and Transmissometry. Puede determinar in-situ tamaños de sedimentos suspendidos: Diámetro Medio Sauter del total de la muestra (SMDt) y de la fracción de gruesos, concentración del total de sedimentos suspendidos y de gruesos, profundidad a la que se efectúa la medición y porcentaje de transmisión óptica (TO). Puede operar en el rango de concentraciones de 0.1 a $1000 \mathrm{mg} / \mathrm{L}$, detectar diámetros medios en el intervalo $2.50-500 \mu \mathrm{m}$ y de 63 a $500 \mu \mathrm{m}$ para gruesos, con rango de TO entre 30 y 95\% (Sequoia, 2009).

El SMDt representa el promedio del diámetro Sauter (SD) del total de las partículas en suspensión. SD se define como el diámetro de una esfera que tiene la misma relación volumen/área superficial que la partícula en consideración. $\mathrm{SD}=\mathrm{d}_{32}=6 \mathrm{~V}_{\mathrm{p}} / \mathrm{A}_{\mathrm{p}}$, dónde $\mathrm{V}_{\mathrm{p}} \mathrm{y} \mathrm{A}_{\mathrm{p}}$ son el volumen y el área superficial de la partícula, respectivamente. En caso de existir floculación, la sonda registra los SMDt de los agregados, en tal situación se calculó el $\mathrm{d}_{50}$ de los agregados con la fórmula $\mathrm{d}_{50}=3.4 \mathrm{SMDt}^{0.83}$ que fuera obtenida para partículas(Filippa et al., 2011).

\section{Caracterización de los sedimentos}

Los sedimentos utilizados en los ensayos corresponden a una muestra del río Salado, recolectada en una sección transversal cercana al camping de la ciudad de Esperanza (31.384134 S, 60.890158 O).

Su caracterización granulométrica se realizó con un difractómetroMalvernMastersizer (2000) en el Laboratorio de Dinamica de Sedimentos Cohesivos (LDSC) - Universidad Federal de Rio de Janeiro (UFRJ).Los resultados indicaron que la muestraposeeun $97 \%$ de finos $(<62.5 \mu \mathrm{m})$ de los cuáles el $85 \%$ fueronlimos y el $11 \%$ arcillas $(<2$ $\mu \mathrm{m})$. A su vez el $65 \%$ de los limos fueron finos $(2-$ $20 \mu \mathrm{m})$. La mediana de la distribución granulométrica (Figura 4) fue $\mathrm{d}_{50}=11.6 \mu \mathrm{m} \quad \mathrm{y}$ $\mathrm{SMDt}=4.88 \mu \mathrm{m}$.

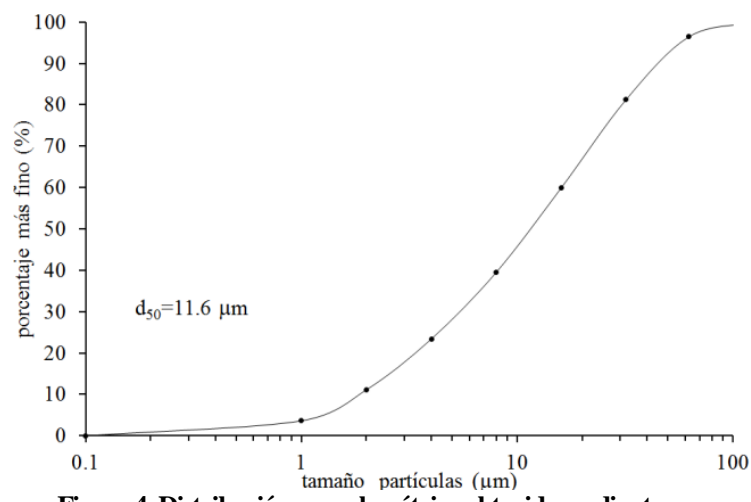

Figura 4. Distribución granulométrica obtenida mediante un difractómetroMalvern de una muestra de sedimentos del río Salado.

La mineralogía de las partículas es un factor relevante a considerar en referencia a la cohesión de los sedimentos suspendidos. En particular, según su composición,las arcillas presentan esta cualidad.Su tendencia a la agregación es mayor para las esmectitas, seguidas por las cloritasy, con la menor capacidad de cohesión las illitas y las caolinitas, (MehtayMcAnally, 2007). 
La composición mineralógica de la muestra de las arcillas empleadas en el reactor fue: $60 \%$ de illita, $30 \%$ deesmectita y 10\% de caolinita (Filippa, 2014). El análisis se realizó por difractometría de rayos $\mathrm{X}$, en el Laboratorio de Mineralogía y Micromorfología del Instituto de Suelos del INTA-CIRN. Esta composición es un fuerte indicativo de la capacidad de floculación de estas arcillas, de acuerdo a MehtayMcAnally (2007).

\section{Metodología de laboratorio}

La primera etapa de los experimentos consistió en la determinación de la hidrodinámica dentro del reactor para un rango de velocidades $\mathrm{N}$, entre 100 y $200 \mathrm{rpm}$. Para ello, se introdujeron 6 litros de agua destilada y se utilizó la sonda FlowTracker para medir la velocidad radial $\left(\mathrm{V}_{\mathrm{r}}\right)$ y tangencial $\left(\mathrm{V}_{\mathrm{t}}\right)$ en distintos radios $\mathrm{r}=5.25$, $6.25,7.25,8.25,9.25$ y $9.75 \mathrm{~cm}$, todos medidos desde el centro del reactor y sobre cuatro planos establecidos a diferentes alturas $Z=10,12,15$ y $18 \mathrm{~cm}$ desde la base del reactor. Estas mediciones fueron comparadas con valores obtenidos de expresiones teóricas (Nagata, 1975) que relacionan $\mathrm{V}_{\mathrm{t}}$ con variables geométricas del reactor, altura de agua inicial $\left(\mathrm{Z}_{2}\right)$, altura del vórtice $(\mathrm{Zo})$, y velocidad angular $(\omega)$. Lavelocidad tangencial teórica, $\mathrm{V}_{\mathrm{t}}$, se obtuvo a partir de las expresiones (1) y (2):

$$
\begin{array}{ll}
\text { Si } r \leq r_{c} & V_{t}=r \omega(1) \\
\text { Si } r \geq r_{c} & V_{t}=\left(\omega r_{c}^{2}\right) / r
\end{array}
$$

Donde $r_{c}($ Figura 2) representa el radio para el cual la masa líquida se mueve como un cilindro sólido y la $\mathrm{V}_{\mathrm{t}}$ sólo depende de $\omega$ y r.El valor de $\mathrm{r}_{\mathrm{c}}$ se determinó utilizando la ecuación (3):

$r_{c}=\sqrt{r_{2}\left(r_{2}-\sqrt{r_{2}^{2}-k}\right)}, \operatorname{conk}=\frac{2 g}{\omega^{2}}\left(Z_{2}-Z_{o}\right)$

Con $\mathrm{k}$ un parámetro de cálculo $\left(\mathrm{m}^{2}\right)$, g la aceleración de la gravedad $\left(9.8 \mathrm{~m} / \mathrm{seg}^{2}\right), \mathrm{Z}_{2}$ la altura de agua medida sobre la pared del cilindro para una determinada $\omega(\mathrm{rad} / \mathrm{s}), Z$ Zo la altura del vórtice $(\mathrm{m})$, $\mathrm{r}_{2} \mathrm{el}$ radio del reactor $(\mathrm{m})$.

Para caracterizar la hidrodinámica,a partir de Bouyer et al. (2005) yCoufort et al. (2008), se calculó la tensión de corte media con la ecuación (4): $\sigma=\rho_{\mathrm{w}} v \sqrt{\frac{0.63}{2}} \mathrm{G}$

Donde $\rho_{\mathrm{w}}$ es la densidad del agua $\left(\mathrm{kg} / \mathrm{m}^{3}\right)$, v es la viscosidad cinemática $\left(\mathrm{m}^{2} / \mathrm{s}\right)$ y $\mathrm{G}(1 / \mathrm{s})$ el parámetro de disipación que se determina con la expresión (5):

$\mathrm{G}=\sqrt{\frac{\mathrm{N}_{\mathrm{p}} \mathrm{d}^{5} \mathrm{n}^{3}}{\mathrm{Vv}}}$

G se relaciona con la potencia disipada en cada jarra por unidad de volumen $\mathrm{V}\left(\mathrm{m}^{3}\right)$, $\mathrm{Np}$ es el número de potencia, d (m) es la longitud de la paleta $\mathrm{yn}=\omega / 60$. Np según Nagata (1975):

$\mathrm{N}_{\mathrm{p}}=\frac{\mathrm{A}}{\operatorname{Re}}+\mathrm{BL}\left(\frac{\mathrm{H}}{\mathrm{D}}\right)^{(0.35+\mathrm{b} / \mathrm{D})}(\operatorname{sen} \theta)^{1.2}$

Donde L se calcula con la expresión (7):

$L=\left(\frac{10^{3}+1.2 \operatorname{Re}^{0.66}}{10^{3}+3.2 \operatorname{Re}^{0.66}}\right)^{\mathrm{p}}$

Con Re el número de Reynolds (ecuación 8), D: diámetro del reactor, $\theta$ : ángulo de las paletas respecto al plano horizontal, b: altura de las paletas y los coeficientes A, B y p definidos por las expresiones (9), (10) y (11) respectivamente:

$\operatorname{Re}=\frac{d^{2} n}{v}$

$A=14+(b / D)\left[670(d / D-0.6)^{2}+185\right]$

$B=10^{\left(1.3-4(\mathrm{~b} / \mathrm{D}-0.5)^{2}-1.14(\mathrm{~d} / \mathrm{D})\right)}$

$\mathrm{p}=1.1+4(\mathrm{~b} / \mathrm{D})-2.5(\mathrm{~d} / \mathrm{D}-0.5)^{2}-7(\mathrm{~d} / \mathrm{D})^{4}$

En la segunda etapa de ensayos se prepararon concentraciones de SST dentro del reactor que cubren el rango observado en el río Salado en condiciones de aguas medias y bajas: 50, 100, 150,300 y $600 \mathrm{mg} / \mathrm{L}$. Para ello, se pesaron cantidades conocidas de los sedimentos del Salado en una balanza analítica (Metler Toledo AB2405/FACT) del laboratorio de Limnología Física (INALI - CONICET, Santa Fe). Con el fin de verificar que las concentraciones de sólidos suspendidos totales observados o medidos (SSTo) en las inmediaciones de los sensores de las sondas fueran representativas de la concentración preparada (SSTp) se tomaron muestras de la 
mezcla agua-sedimentos dentro del reactor. Las mismas se extrajeron por vacío, mediante un tubo de goma de $0.5 \mathrm{~cm}$ de diámetro, ubicado a $15 \mathrm{~cm}$ del fondo del reactor y a $7.25 \mathrm{~cm}$ del centro del mismo(denominado punto $\mathrm{M}$ ). Este punto de medición $\mathrm{M}$ se seleccionó teniendo en cuenta las siguientes características: i) en él se disponían valores de velocidad tangencial y radial medidas, ii) M no era afectado por el vórtice para el rango de rpm evaluados, es decir, los sensores siempre se encontraban sumergidos, iii) era un punto de perturbación mínimade la hidrodinámica del sistema,al sumergir los sensores.

Se recolectaron muestras de $100 \mathrm{~mL}, 200 \mathrm{~mL}$ y $500 \mathrm{~mL}$. Las concentraciones se determinaron con el método de filtrado, según la norma ASTM D3977-97 (Guo, 2006), utilizando filtros Millipore de $47 \mathrm{~mm}$ de diámetro y $0.45 \mu \mathrm{m}$ de poro y la balanza analítica.

Las etapas posteriores de los ensayos consistieron en la medición de la turbiedad $(\mathrm{T})$ de la mezcla agua-sedimento dentro del reactor con la sonda YSI 6000. Las determinaciones se realizaron para variaciones de las diferentes variables involucradas, a saber: variaciones de SSTp en el rango ya mencionado, diferentes alturas de los sensores medidas desde la base del reactor $(Z=8$, 15 y $21 \mathrm{~cm}$ ), distintos radios medidos desde el centro del reactor $(\mathrm{r}=0,3.75,5.75$ y $7.75 \mathrm{~cm})$ ydistintas velocidades angulares $\mathrm{N}=100,125,150$, 175 y $200 \mathrm{rpm}$. Estas determinaciones se realizaron para verificar la homogeneidad de la mezcla.

La medición del SMDtde las distintas mezclas preparadas se realizó con la sonda LISST-25Xen la posición del punto $\mathrm{M}$.

Por último, se efectuaron mediciones de la mezcla de sedimentos finos con agua destilada adicionando una concentración predeterminada de $20 \mu \mathrm{g} / \mathrm{L}$ de Amidorodamina $\mathrm{G}$ extra. Este trazador cumple con una serie de características, necesarias para el tipo de experiencia realizada. Smart et al. (1977) analizaron el comportamiento de 8 trazadores fluorescentes, entre ellos la Amidorodamina $G$ extra, cuyas características más sobresalientes son:1) la estabilidad de la fluorescencia a valores de $\mathrm{pH}$ comprendidos entre 6 y 10,2) no presentar cambios sensibles de fluorescencia ante variaciones importantes de temperatura $\left(-25\right.$ a $\left.\left.25{ }^{\circ} \mathrm{C}\right), 3\right)$ tener bajas tasas de fotodecaimiento (tanto por luz solar como artificial),4)resistencia a la absorción, tanto de la materia orgánica como inorgánica,5)no ser tóxico para la salud humana y el medio ambiente (Behrens et al., 2001).

\section{RESULTADOS Y DISCUSIÓN}

\section{Análisis de las condiciones hidrodinámicas}

En las Figuras 5 y 6 se presentan a modo de ejemplo los valores de las velocidades tangenciales medidas $\left(V_{t}\right)$ en $\mathrm{Z}=10,12,15$ y $18 \mathrm{~cm}$ y las calculadas $\left(V_{t^{\prime}}\right)$ con la expresión (2). Se advierte que los valores medidos presentan pequeñas variaciones en sentido radial (de hasta un $15 \%$ entre la máxima y la mínima) en tanto que, en sentido vertical, las variaciones no son importantes.

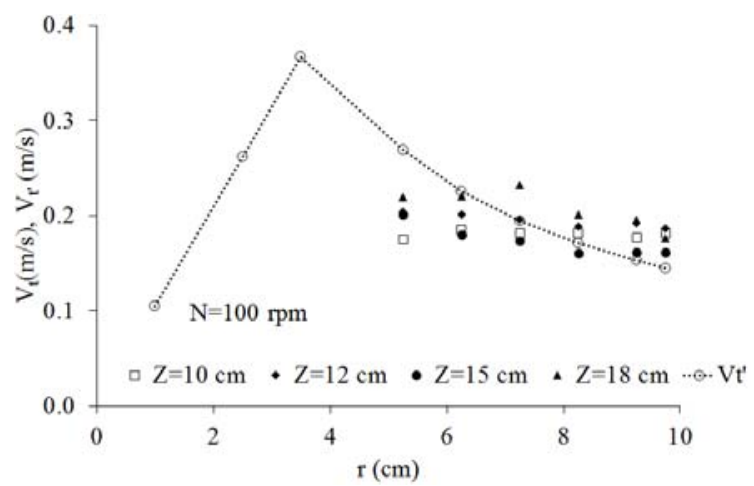

Figura 5: Velocidades tangenciales medidas $\left(V_{t}\right)$ vs calculadas $\left(V_{t^{\prime}}\right)$, en $Z=10,12,15$ y $18 \mathrm{~cm}$. $N=100$ rpm

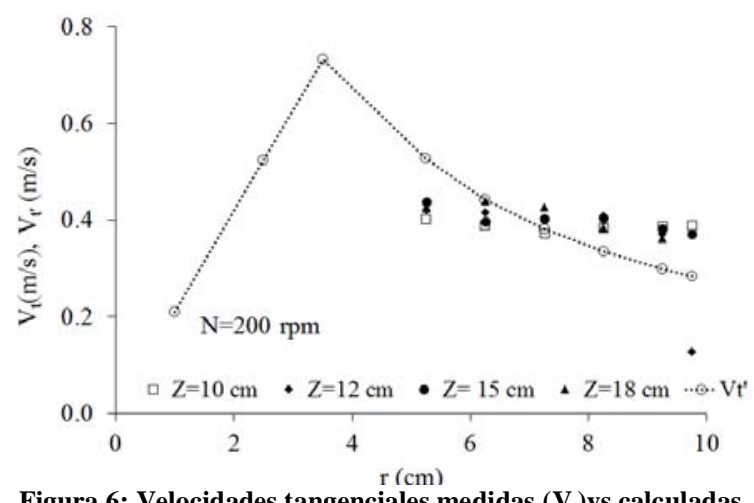

Figura 6: Velocidades tangenciales medidas $\left(V_{t}\right)$ vs calculadas $\left(V_{t}\right)$, en $Z=10,12,15$ y $18 \mathrm{~cm}$. $N=200 \mathrm{rpm}$

Teniendo en cuenta estos resultados se obtuvieron las $\mathrm{V}_{\mathrm{t}}$ promediadas en la vertical, en $\mathrm{m} / \mathrm{s}$, Tabla 1.

El valor de $\mathrm{V}_{\mathrm{t}}$ en el punto de medición $\mathrm{M}(\mathrm{r}=7.25$ $\mathrm{cm}, Z=15 \mathrm{~cm}$ ), varía entre 0.20 y $0.40 \mathrm{~m} / \mathrm{s}$. Los valores medidos y calculados guardan similitud en magnitud y tendencia, pero se evidencian diferencias en la zona cercana a la pared del reactor $(\mathrm{r}=9.75 \mathrm{~cm})$ y en $\mathrm{r}=5.25 \mathrm{~cm}$, el más cercano a $\mathrm{r}_{\mathrm{c}}$. Los valores de $r_{c}$ obtenidos para todas las $\mathrm{N}$ analizadas, estuvieron 
en el rango de 3.6 a $3.9 \mathrm{~cm}$.Se observó que para el cálculo de $r_{c}$, la variable que presentó mayor incertidumbre en la medición fue Zo, debido a la inestabilidad que se presentó en el vórtice a medida que aumentó $\mathrm{N}$.

Tabla 1. Variación radial de $V_{t}$ promediadas en la vertical $(\mathrm{m} / \mathrm{s})$, para $\mathrm{N}=100,135,156$ y $200 \mathrm{rpm}$

\begin{tabular}{|c|c|c|c|c|c|c|}
\hline \multirow{2}{*}{$\begin{array}{c}\mathbf{N} \\
(\mathbf{r p m})\end{array}$} & \multicolumn{6}{|c|}{$\mathbf{r}(\mathbf{c m})$} \\
\cline { 2 - 7 } & $\mathbf{5 . 2 5}$ & $\mathbf{6 . 2 5}$ & $\mathbf{7 . 2 5}$ & $\mathbf{8 . 2 5}$ & $\mathbf{9 . 2 5}$ & $\mathbf{9 . 7 5}$ \\
\hline $\mathbf{1 0 0}$ & 0.20 & 0.20 & 0.20 & 0.18 & 0.18 & 0.18 \\
\hline $\mathbf{1 3 5}$ & 0.26 & 0.26 & 0.25 & 0.24 & 0.25 & 0.23 \\
\hline $\mathbf{1 5 6}$ & 0.33 & 0.32 & 0.32 & 0.30 & 0.29 & 0.25 \\
\hline $\mathbf{2 0 0}$ & 0.42 & 0.41 & 0.40 & 0.39 & 0.38 & 0.30 \\
\hline
\end{tabular}

Por su parte, las $\mathrm{V}_{\mathrm{r}}$, variaron entre 0.02 y $0.08 \mathrm{~m} / \mathrm{s}$ para el rango de $\mathrm{N}$ analizado; es decir un orden de magnitud inferior a las $\mathrm{V}_{\mathrm{t}}$. Además, se registraron variaciones en sentido vertical y radial, siendo estas últimas las más significativas, donde, cerca de la pared, la $\mathrm{V}_{\mathrm{r}}$ promedió valores del orden de los $0.02 \mathrm{~m} / \mathrm{s}$, mientras que cerca del vórtice fueron de $0.06 \mathrm{~m} / \mathrm{s}$.

Los valores de Re estuvieron en el rango de $1.710^{3}$ $3.310^{4}$, lo que confirma el régimen turbulento $(\mathrm{Re}>1000$ régimen turbulento,Nagata, 1975) de los experimentos. Las tensiones de corte, $\sigma$, calculadas de acuerdo a la ecuación 4 (Nagata, 1975), estuvieron en el intervalo $0.038<\sigma<0.098 \mathrm{~N} / \mathrm{m}^{2}$, compatibles con las tensiones calculadas para el río Salado en aguas bajas y medias (Trento y Alvarez, 2011), al igual que el parámetro $\mathrm{G}$, en el intervalo $68<\mathrm{G}<1751 / \mathrm{s}$, para 100 y $200 \mathrm{rpm}$ respectivamente.

\section{Relación de las SSTp y SSTo. Verificación de la homogeneidad de la mezcla}

En la Figura 7se graficaron los valores de SSTp vs SSTo para los volúmenes de muestreo de 100, 200 y 500 $\mathrm{mL}$.Todas las muestras fueron tomadas en el punto $\mathrm{M}$. La línea de trazos a $45^{\circ}$ representa $\mathrm{SSTp}=\mathrm{SST}$.

Se puede observar que a medida que aumenta el volumen de muestreo las SSTp se aproximan a las SSTo, de este modo la curva para el volumen de 500 mLes la más próxima a la línea de $45^{\circ}$. De tal modo, los registros de turbiedad obtenidos con la sonda fueron compatibles con las SSTp específicas para cada experimento, poniendo en evidencia la homogeneidad de la mezcla.

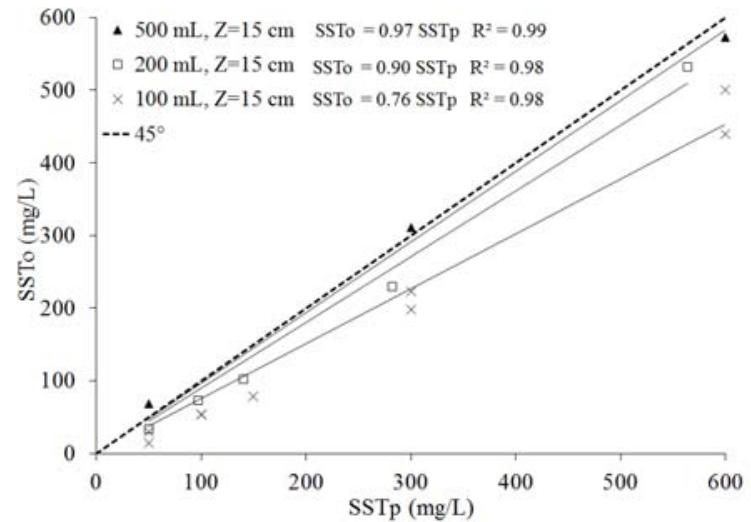

Figura 7: Concentraciones preparadas vs observadas. SSTp vs SSTo

A la vez se cuantificó el error debido a los procedimientos de filtrado de las muestras en laboratorio para obtener SSTo. Este error se debe básicamente a las acciones de filtrado, secado y pesado. Para ello se realizó el análisis para 6 concentraciones distintas en el intervalo $100<\mathrm{SSTp}<600 \mathrm{mg} / \mathrm{L}$, todas con un volumen de $200 \mathrm{~mL}$. El error relativo entre las SSTp vs SSTo, estuvo en el intervalo 3.1\%-7\%. Los 6 errores fueron negativos, es decir, la determinación fue por defecto y su magnitud independiente de las concentraciones.

\section{Análisis de la variación de la turbiedad (T)}

Se realizaron mediciones de $\mathrm{T}$ con la sonda YSI 6600 para variaciones de N, SSTp, r y Z. En la Figura 8 se muestran las mediciones realizadas para las concentraciones de 300 y $600 \mathrm{mg} / \mathrm{L}$, para diferentes radios (r) medidos desde el centro del reactor y para diferentes alturas $(\mathrm{Z})$ medidas desde el fondo, para $\mathrm{N}=100 \mathrm{rpm}$.

Para ambas SSTp se observaron diferencias positivas de $\mathrm{T}$ de no más de $10 \mathrm{NTU}$, tanto en sentido vertical como radial.

En la Figura 9 se presentan las mediciones de $\mathrm{T}$ efectuadas para diferentes concentraciones $\mathrm{y}$ variaciones de la velocidad angular de la paleta $(\mathrm{N})$ en el rango 100-200 rpm, en ausencia de rodamina. Se observa que independientemente de las SSTp, se incrementó la turbiedad a medida que aumentó $\mathrm{N}$, un $15 \%$ en promedio. Los valores de $\mathrm{T}$ para $\mathrm{N}=200 \mathrm{rpm}$ fueron mayores a los de $100 \mathrm{rpm}$ para todas las SSTp. 


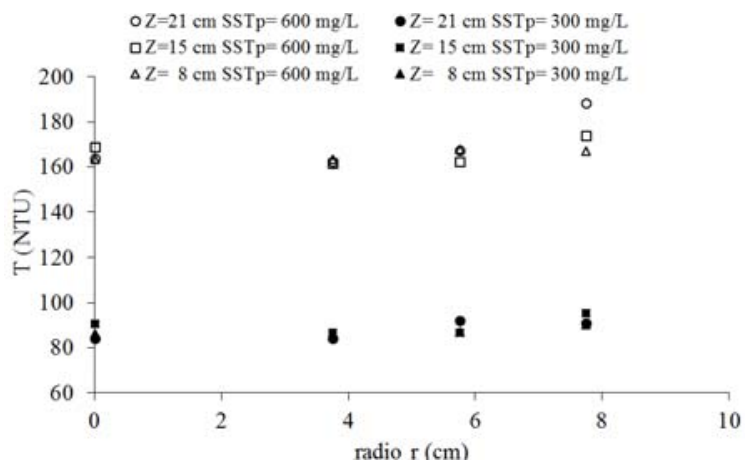

Figura 8: Mediciones de turbiedad para SSTp=300y 600 $\mathrm{mg} / \mathrm{L}$, en diferentes $\mathbf{r}$ medidos desde el centro y distintas Zmedidas desde el fondo del reactor. $\mathrm{N}=100 \mathrm{rpm}$.

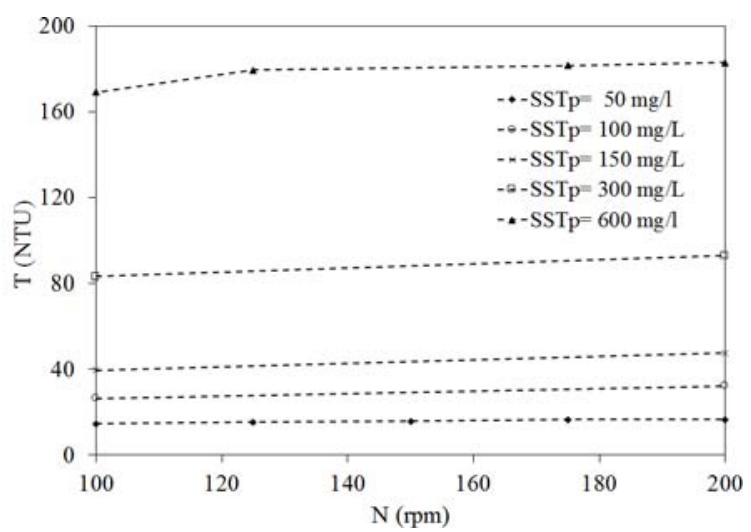

Figura 9: Mediciones de turbiedad para distintas SSTpy velocidades angulares $(\mathrm{N})$, sin rodamina.

Las variaciones de T para distintas SSTp medidas en el reactorcon rodamina (línea de trazos) y sin rodamina (línea llena) se presentan en la Figura 10. Se ajustaron funciones lineales para las medicionescorrespondientes aN=200 y $100 \mathrm{rpm}$. Se observa que la presencia de rodamina produce un descenso de la turbiedad para todas las SSTp, lo cual queda en evidencia por las pendientes de las rectas de ajuste.Para $\mathrm{N}=200 \mathrm{rpm}$ la disminución de la turbiedad es de $45 \%$, para SSTp $=50 \mathrm{mg} / \mathrm{L}$ y continúa con 24,11 y $9 \%$ para $S S T p=150,300$ y $600 \mathrm{mg} / \mathrm{L}$ respectivamente.Para $\mathrm{N}=100 \mathrm{rpm}$ también la rodamina produce una disminución de $\mathrm{T}$, en el intervalo $6 \%-18 \%$, con un $15 \%$ para SSTp $=50 \mathrm{mg} / \mathrm{L}$.

En la Figura 11 se muestran las concentraciones de rodamina para las diferentes SSTp y N ensayadas. Se advierte que se mantienen prácticamente constantes, en torno a un valor medio de $21.3 \mathrm{mg} / \mathrm{L}$ y con un intervalo de desvíos 20.5-22.2 $\mathrm{mg} / \mathrm{L}$, para todo el rango de N y SSTp. Es decir, que T aumenta con las concentraciones en forma independiente de la presencia del trazador, para cualquier $\mathrm{N}$.
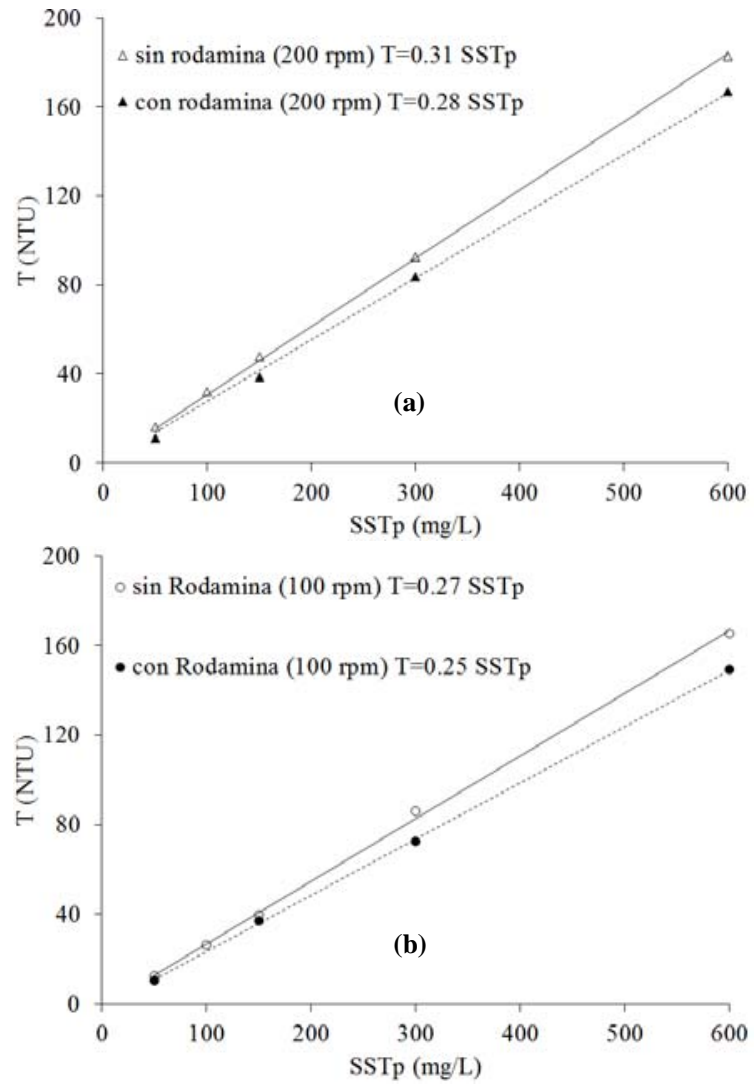

Figura 10: Mediciones de turbiedad,con y sin rodamina, para variaciones de SSTp. (a) $\mathrm{N}=200 \mathrm{rpm}$ y (b) $\mathrm{N}=100 \mathrm{rpm}$

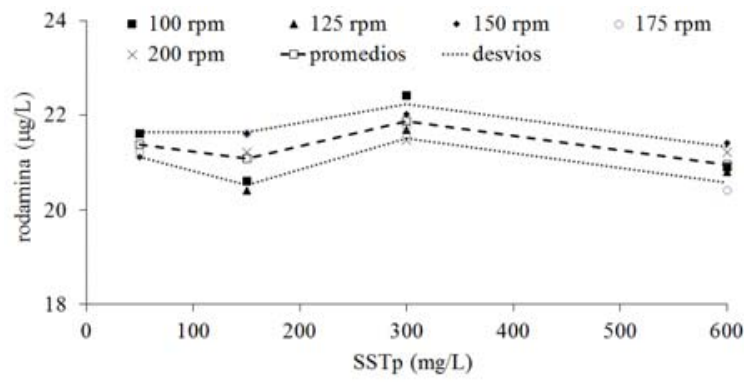

Figura 11: Mediciones de concentraciones de rodamina para variaciones de SSTp y $\mathbf{N}$.

\section{Análisis del diámetro de los flóculos: $\mathbf{d f}_{50}$}

En la Figura 12se graficaron los valores de $\mathrm{df}_{50}$, obtenidos con la sonda LISST, para distintas $\mathrm{N}$ y distintas SSTp, en ensayos con y sin rodamina. Se advierte que los máximos $\mathrm{df}_{50} \approx 48 \mu \mathrm{m}$ se obtienen sin rodamina, para SSTpentre 50 y $100 \mathrm{mg} / \mathrm{L}$ y N superiores a $150 \mathrm{rpm}$, es decir con las mayores tensiones de corte. Para valores mayores de SSTp, los $\mathrm{df}_{50}$ decaen para todo el rango de $\mathrm{N}$ hasta $\mathrm{df}_{50} \approx 25 \mu \mathrm{m}$, independientemente de la presencia de 
rodamina.Puede inferirse que el aumento de tensiones de corte para bajas SSTp aumenta la frecuencia de colisiones entre partículas, de modo tal que el resultado final es la producción de microflóculos de hasta $48 \mu \mathrm{m}$ y menor turbiedad, producto de la agregación. Mientras que para SSTp mayores y por consiguiente con una presencia de más partículas de sedimentos, las mismas tensiones redundan en la formación de microflóculos más pequeños y una mayor turbiedad. Este tipo de proceso de agregación y desagregación fue esquematizado por Dyer (1989) mediante su conocido diagrama conceptual.

Para una concentración constante de rodamina de 20 $\mu \mathrm{g} /$ Len el rango inferior de SSTp, para las tensiones intermedias empleadas, se obtuvo un tamaño máximo $\mathrm{df}_{50} \approx 35 \mu \mathrm{m}$. Para $\mathrm{SSTp}>150 \mathrm{mg} / \mathrm{L}$ con rodamina, los $\mathrm{df}_{50}$ superaron entre 5 y $10 \%$ a los obtenidos sin rodamina. Las TO estuvieron en el rango $13 \%-83 \%$, es decir que todas las determinaciones de $\mathrm{df}_{50}$ estuvieron dentro de los límites admisibles del equipo LISST-25X.

Sedebetenerencuentaquelafloculacióngeneralmenteo curreaescalacercanaaladeKolmogorov(Coufortetal.,2 008),lacualdefineeltamañodelosremolinosmáspequeñ os, $\eta=\left(v^{3} / \varepsilon\right)^{1 / 4}$, quedisipanlaenergíadelaturbulencia,co nelatasadedisipaciónviscosadelaenergíacinética.Para estosexperimentossecalculó $=121 \mu$ mparaN=100rpm $\mathrm{y} \eta=76 \mu \mathrm{mparaN}=200 \mathrm{rpm}$.Entodosloscasoslostamaño smediosdelosagregadosfueronmenoresa $\rceil$,pudiéndos ededucirentoncescondicionesdeturbulenciaisotrópica enelreactor.

\section{CONCLUSIONES}

Se realizaron mediciones de velocidad tangencial -y radial- y se lograron ajustes satisfactorios con las expresiones teóricas de $\mathrm{V}_{\mathrm{t}}$, destacándose velocidades en la zona de medición de 0.20 a $0.40 \mathrm{~m} / \mathrm{s}$. El número de Re estuvo en el rango1.7 $10^{3}$ y $3.310^{4}$, lo que indica régimen turbulento, mientras que las tensiones de corte y los gradientes de velocidad estuvieron en los rangos de $0.038-0.098 \mathrm{~N} / \mathrm{m}^{2}$ y de 68-175 1/s, para 100 y $200 \mathrm{rpm}$ respectivamente.

Se determinó que T aumenta con las SSTp en forma independiente de la presencia del trazador, para cualquier $\mathrm{N}$.

En ausencia de rodamina la turbiedad fue mayor (15\% en promedio) a medida que aumentó $\mathrm{N}$, independientemente de las SSTp. Los valores de T para $\mathrm{N}=200 \mathrm{rpm}$ fueron mayores a los $\mathrm{T}$ para $100 \mathrm{rpm}$ para todas las SSTp. Para iguales condiciones generales, la presencia de rodamina produjo un descenso de la turbiedad para todas las SSTp. La disminución fue de $45 \%$, para $\mathrm{SSTp}=50 \mathrm{mg} / \mathrm{L}$ y continuó con 24, 11 y 9\% para $\mathrm{SSTp}=150,300$ y 600 $\mathrm{mg} / \mathrm{L}$ respectivamente. Para $\mathrm{N}=100 \mathrm{rpm}$ también la rodamina produjo una disminución de $\mathrm{T}$, en el intervalo $6 \%-18 \%$, con un $15 \%$ para $\mathrm{SSTp}=50 \mathrm{mg} / \mathrm{L}$.

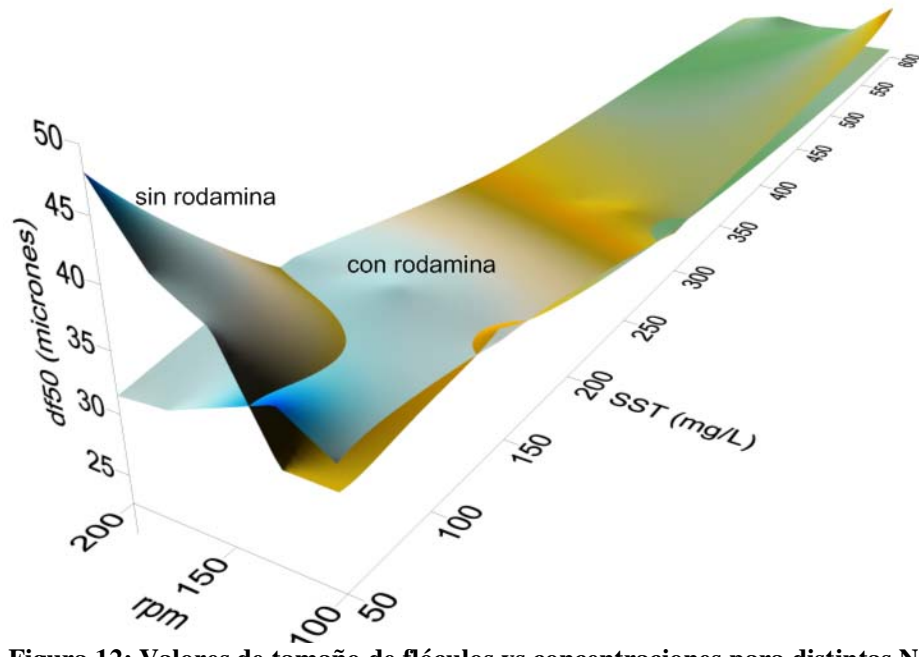

Figura 12: Valores de tamaño de flóculos vs concentraciones para distintas $\mathbf{N}$

Los máximos tamaños promedio de flóculos, $\mathrm{df}_{50} \approx 48 \mu \mathrm{m}$, se obtuvieron sin rodamina, para SSTp entre 50 y $100 \mathrm{mg} / \mathrm{L}$ y $\mathrm{N}$ superiores a $150 \mathrm{rpm}$, es decir con las mayores tensiones de corte y la menor turbiedad. Para valores mayores de SSTp, los $\mathrm{df}_{50}$ decayeron para todo el rango de $\mathrm{N}$ hasta $\mathrm{df}_{50} \approx 25 \mu \mathrm{m}$, 
independientemente de la presencia de rodamina. Para SSTp $>150 \mathrm{mg} / \mathrm{L}$ con rodamina, $\operatorname{los} \mathrm{df}_{50}$ fueron entre 5 y $10 \%$ mayores a los obtenidos sin rodamina.

Se concluye que el aumento de tensiones de corte para bajas SSTpredunda en la producción de microflóculos más grandes y con menor turbiedad. Mientras que para SSTp mayores las mismas tensiones generan microflóculos más pequeños y una mayor turbiedad.

Se realizaron avances importantes sobre la interacción trazador-sedimentos para diferentes condiciones hidrodinámicas en un ambiente controlado, y se encuentran en etapa desarrollo una serie de ensayos, perobajo otras condiciones hidroambientales que permitirán una comprensión más acabada del fenómeno.

\section{AGRADECIMIENTOS}

Este trabajo fue subsidiado por los Proyectos de Investigación CAI+D 2011 PI 50120110100326 y PIC 50420150100048LI 2016 subsidiados por la Universidad Nacional del Litoral (UNL-Santa Fe).

\section{REFERENCIAS BIBLIOGRAFICAS}

Behrens, H., Beims, U., Dieter, H., Dietze, G., Eikmann, T., Grummt, T., Hanisch, H., Henseling, H., Käß, W., Kerndorff, H., Leibundgut, C., MüllerWegener, U., Rönnefahrt, I., Scharenberg, B., Schleyer, R., Schloz, W. y Tilkes, F. (2001) Toxicological and EcotoxicologicalAssessment of WaterTracers, Hydrogeology Journal, 9(3):321-325. doi: $10.1007 / \mathrm{s} 100400100126$

Benevides, X., Oliveira Silva, I.,GallisaGuimarães L., Gallo, M., Parente Ribeiro, C. yFigueiredo, A. (2014) Estimation of suspended sediment concentration by acoustic scattering: an experimental and theoretical analysis for spherical particles.J Soils Sediments, 14, 1325-1333. doi: 10.1007/s11368014-0905-5

Bertrand, M., Parmentier, D., Lebaigue, O., Plasari, E. yDucros F. (2012) Mixing Study in an Unbaffled Stirred Precipitator Using LES Modelling. International Journal of Chemical Engineering, ID 450491, 11 pages.doi:10.1155/2012/450491

Bouyer, D., Coufort, C., Liné, A., Do-Quang, Z. (2005) Experimental analysis of floc size distributions in a 1-L jar under different hydrodynamics and physicochemical conditions, Journal of Colloid and Interface Science, 292 (2) 413-428.doi: 10.1016/j.jcis.2005.06.011

Carr, M. y Rehmann, C. (2007) Measuring the Dispersion Coefficient with Acoustic Doppler Current Profilers.Journal of Hydraulic Engineering, 133(8).doi:10.1061/(ASCE)07339429

Chapra, S.C. (1997) Surface Water-Quality Modelling. John Wiley and Sons Inc., Iowa, 844 p.

Cheng, W.P., Kao, Y.P. y Yu, R. F. (2008)A novel method for on-line evaluation of floc size in coagulation process.Water Research, 42 (10-11), 2691-2697.doi: 10.1016/j.watres.2008.01.032

Clark, D., Feddersen, F., Omand, M. yGuza R. (2009)Measuring Fluorescent Dye in the Bubbly and Sediment-Laden Surfzone.Water, Air and Soil Pollution, 204 (1-4), 103-115. doi: 10.1007/s11270-009-0030-Z

Coufort, C., Dumas, D., Bouyer, D. yLiné, A. (2008) Analysis of floc size distributions in a mixing tank.Chemical Engineering and Processing, 47(3), 287-294. doi: 10.1016/j.cep.2007.01.009

Dyer, K. R. (1989) Sediment Processes in estuaries: Future research requeriments.Journal of Geophysical Research, 94, C10, 14327-14339. doi: 10.1029/JC094iC10p14327

Edzwald, J.K., Upchurch, J.B. yO'Melia, C.R. (1974) Coagulation in Estuaries.Environmental Science and Technology, 8 (1) 58-63.

Filippa, L., Freire, L., Trento, A., Alvarez, A., Gallo, M. y Vinzón, S. (2011) Laboratory evaluation of two LISST-25X using river sediments.SedimentaryGeology, 238, 268-276. doi: 10.1016/j.sedgeo.2011.04.017

Filippa, L. (2014) Estudio de la dinámica de la floculación y su relación con el transporte de cromo en el río Salado (Santa Fe). (Tesis de Doctorado), Facultad de Ingeniería y Ciencias Hídricas, Universidad NAcional del Litoral, Santa $\mathrm{Fe}$, Argentina.

Fischer, H.B. (1968) Dispersion predictions in natural streams.Journal of the Sanitary EngineeringDivision, ASCE, 94 (5), 927-943.

Fischer,H. B., List, E. J., Koh, R. C. Y., Imberger, J. y Brooks, N. H.. (1979)Mixing in inland and coastalwaters. AcademicPress.

Goes, Y.W., Buraschi, G.B., Freire, L.C., Benevidez, C.X., Álvarez, A. M. T., Gallo, M.N., Gallisa, L. (2019) Mediciones de concentración con sondas de turbiedad (OBS) en la presencia de mezclas de sedimentos. VI Simposio sobre métodos experimentales en hidráulica. Paysandú, República

Oriental del Uruguay. 
Guo, Q. (2006) Correlation of Total Suspended Solids (TSS) and Suspended Sediment Concentration (SSC) Test Methods.New Jersey Department of Environmental Protection, Division of Science, Research and Technology. Contract No. SR05-005.

Hamidifar, H., Omid, M. yKeshavarzi, A. (2015)Longitudinal dispersion in waterways with vegetated floodplain.Ecological Engineering, 84, 398-407. doi: 10.1016/j.ecoleng.2015.09.048

Liu H. (1977) Predicting Dispersion Coefficient of Streams.Journal of the Environmental Engineering, 103(1), 59-69.

Mehta, A. yMcAnally, W. W. (2007) Fine-grained sediment transport. En M. Garcia.(Ed.), Sedimentation Engineering Processes, Measurements, Modeling, and Practice (pp. 253306).Reston, Estados Unidos de Norteamérica: American Society of Civil Engineers.

Mikes, D., Verney, R., Lafite, R. y Belorgey, M. (2004) Controlling factors in estuarine flocculation processes experimental results with material from the Seine Estuary, Northwestern France. Journal of Coastal Research, Special Issue 41.

Nagata, S. (1994) Mixing: principles and applications.Chichester, Halsted Press Wiley.

Rutherford, J.C. (1994) River mixing. New York. John Wiley and Sons.

Sequoia (2009) LISST-25 User's Guide, 2009.http://sequoiasci.com.

Shen, C., Niu, J., Anderson, E. yPhanikumar, M. (2010) Estimating longitudinal dispersion in rivers using Acoustic Doppler Current Profilers.Advances in Water Resources, 33(6), 615-623.doi: 10.1016/j.advwatres.2010.02.008

Smart, P.L. y Laidlaw, I.M.S. (1977)An Evaluation of Some Fluorescent Dyes for Water Tracing. Water Resources Research, 13(1), 15-33. doi: 10.1029/WR013i001p00015

Sontek (2009) FlowTracker Handheld ADV Technical Manual Firmware 3.7 Software Version 2.30. San Diego, EstadosUnidos de Norteamérica.
Trento, A. yÁlvarez, A.M.T. (2011) A numerical model for the transport of chromium and fine sediments.Environmental Modeling and Assessment, 16(6), 551-564.doi: 10.1007/s10666011-9263-5.

Winterwerp, J.C. (2002) On the flocculation and settling velocity of estuarine mud, Continental Shelf Research, 22(9), 1339-1360. doi: 10.1016/S02784343(02)00010-9

YSI $\quad 6600 \quad$ (2009) User Manual.http://www.ysi.com/media /pdfs/E526600V2.pdf

Zhenbei Whang, J.N., XiaoyuJi, Y. Y. (2018) Effect of the micro-flocculation stage on the flocculation and settling velocity of estuarine mud.Science of the Total Environment, 633(15) 1183-1191. doi: 10.1016/j.scitotenv.2018.03.286

\section{Tipo de Publicación: ARTÍCULO.}

Trabajo recibido el 14/02/2019 y aprobado para su publicación el 30/09/2019.

\section{COMO CITAR}

Wolfenson, A., Lanza, V., Palman, L., Imhoff, M., Alvarez, A. y Trento, A. (2019) Interacción aguasedimentos-trazador fluorescente en un reactor. Medición de flóculos. Cuadernos del CURIHAM. 25 , 19-29.

DOI: https://doi.org/10.35305/curiham.v25i0.114

Este es un artículo de acceso abierto bajo licencia: Creative Commons Atribución - No Comercial Compartir Igual 4.0 Internacional (CC BY-NC-SA 4.0) (https://creativecommons.org/licenses/by-ncsa/4.0/deed.es) 